\title{
Anomaly event detection for sit-to-stand transition recognition to improve mariner physical activity classification during a sea voyage.
}

\author{
Ik-Hyun Youn ${ }^{1}$, Jong-Hoon Youn ${ }^{1}$, Jung-Min Lee ${ }^{2}$, Chol-Seong Kim ${ }^{\text {* }}$ \\ ${ }^{1}$ College of Information Science and Technology, University of Nebraska at Omaha, NE, USA \\ ${ }^{2}$ School of Health, Physical Education and Recreation, University of Nebraska at Omaha, NE, USA \\ ${ }^{3}$ Division of International Maritime Transportation Science, Mokpo National Maritime University, Korea
}

\begin{abstract}
During a sea voyage, seafarers live on an unstable surface that demands unusual physical activity patterns. Previous studies recognized physical inactivity patterns in their special living environment. This study aims to develop a new sit-to-stand transition detection method based on physical activity classification models using wearable sensors to objectively assess the unstable physical activity patterns of seafarers. The sit-to-stand event detection was selected to improve the classification accuracy of the static activities including sitting and standing during the physical inactivity patterns. Anomaly detection was applied to handle imbalanced training data of sit-to-stand events. A single wearable sensor was attached in a chest pocket of the uniform to extract the upper trunk motion features such as body angle and dynamics. Two classification models with and without sit-to-stand event detection were developed and compared with each other to evaluate the efficacy of the sit-to-stand event detection in classifying the mariners' physical activity. Our experimental results showed that the sit-to-stand event detectionbased model significantly improved the classification accuracy of static activities. We expect that the proposed model can be integrated into a continuous physical activity monitoring system for the objective assessment of mariner's physical health in an unstable living environment.
\end{abstract}

Keywords: Seafarer's physical activity classification, Sit-to-stand detection, Imbalance data training, Wearable sensor, Unstable living environment.

Accepted on May 22, 2017

\section{Introduction}

Seafarers live in a special circumstance that is unstable and isolated during a sea voyage. It is important to understand seafarers' unique lifestyle in the tough living conditions to maintain their physical health status [1,2]. Previous studies addressed that physical activity in an unstable living environment reinforced different characteristics of physical activities due to the ship's motion in waves $[3,4]$. For example, walking on a ship at sea required significantly higher muscular efforts than the walking on a stable floor [5]. Unstable ship movement also triggered physical inactivity that was highly prevalent in seafarers [6-8]. It is important to have reliable physical activity assessments to gain a better understanding of seafarers' physical activity patterns from their distinctive living and working environment.

Wearable inertial sensor-based approaches are frequently used physical activities in daily life [9]. Wearable sensor-based physical activity studies have been well investigated in various populations on land [10-12]. However, these studies may not be directly applicable for seafarers. This because wearable approaches for mariners needs to consider additional uncommon conditions such as dynamic ship's motion and limited accommodation area. Due to the seafarer's prevalent sedentary behavior, accurate classification between sitting and standing is more important than other daily activities. The two sedentary activities (i.e. sitting and standing) can be easily misclassified because upper trunk motion characteristics of both activities are fundamentally similar to each other. In this study, we assume that the Sit-To-Stand transition (STS) is a useful indicator for recognizing the transitions between sedentary behaviors (i.e. sitting-to-stand or stand-to-sit). Since the STS event duration is much shorter than that of other major physical activities, we apply an anomaly event detection algorithm to handle the imbalanced training data.

In brief, this paper proposes the STS detection-based seafarer physical activity classification method using a single wearable sensor. We developed two physical activity classification models (with and without the STS detection). The STS detection-based model is compared to a simple classification model without the STS detection. The following sections describe the details of the classification model development steps. 


\section{Methodology}

\section{Data collection}

Nineteen subjects $(n=19)$ participated in the experiment. All participants were apprentices on the training ship of the Mokpo National Maritime University in Korea. Before commencing the experiment, participants provided self-reported characteristics. Detailed information of participants is shown in Table 1.

Table 1. Participant characteristics.

\begin{tabular}{ll}
\hline Characteristics & Mean (Standard deviation) \\
\hline $\mathrm{n}$ & 19 \\
\hline Female/male & $3 / 16$ \\
\hline Age $(\mathrm{y})$ & $22.0(0.7)$ \\
\hline BMl $\left(\mathrm{kg} \cdot \mathrm{m}^{2}\right)$ & $22.9(3.1)$ \\
\hline Height $(\mathrm{cm})$ & $175.1(7.7)$ \\
\hline Weight $(\mathrm{kg})$ & $70.5(13.0)$ \\
\hline
\end{tabular}

*BMI; Body Mass Index.

The accelerometer sensor was placed in the chest pocket of the uniform of participants to avoid interrupt of their ordinary physical activity patterns. In this study, the Shimmer 3 sensor was used that is a low power wireless sensor platform to collect 3-dimensional acceleration [13]. The sampling frequency of the wearable sensor was set at $100 \mathrm{~Hz}$. All subjects conducted about $20 \mathrm{~min}$ of structured activities including lying, sitting, STS, standing, and walking. The detailed activity description is in Table 2 .

\section{Feature extraction}

The trunk orientation and motion of the subject were computed to describe the characteristics of each physical activity. The trunk orientation measures the tilted angle from the horizontal plane. Since the upper trunk can be tilted either anteriorposterior (i.e. pitch angle) or Medio-lateral direction (i.e. roll angle), the largest angle between two angles was taken as the trunk orientation from the gravity direction. The trunk orientation was defined by adapting the Euler angles on the horizontal plane [14].

Table 2. Activities undertaken in the experiment.

\begin{tabular}{lll}
\hline Activity type & Duration (s) & Description \\
\hline Lying & 180 & Lying on back and side \\
\hline Break & 60 & Static with comfortable posture \\
\hline Sitting & 120 & Sitting on a chair \\
\hline Fast STS & 2 & Fast sit-to-stand within 2 s \\
\hline Standing & 120 & Standing still \\
\hline Fast STS & 2 & Fast stand-to-sit within 2 s
\end{tabular}

\begin{tabular}{lll}
\hline Sitting & 120 & Sitting on a chair \\
\hline Slow STS & 2 to 4 & Slow sit-to-stand more than $2 \mathrm{~s}$, less than $4 \mathrm{~s}$ \\
\hline Standing & 120 & Standing still \\
\hline Slow STS & 2 to 4 & Slow stand-to-sit more than $2 \mathrm{~s}$, less than $4 \mathrm{~s}$ \\
\hline Sitting & 60 & Sitting on a chair \\
\hline Break & 60 & Static with comfortable posture \\
\hline Walking & 180 & Walking at comfortable speed \\
\hline Total duration & About 900 & Excluding breaks
\end{tabular}

The Trunk Angle (TA) estimations are shown in Equations 1 and 2. The Equations 1 and 2 are used to estimate the roll and pitch angles from the lateral- and vertical-direction acceleration. The pitch angle is obtained using the Equation 1.

$\theta=\sin ^{-1}\left(a_{\mathrm{x}} / g\right) \rightarrow(1)$

Where $a_{\mathrm{x}}$ is the lateral direction acceleration and $g$ is the gravity. Then, the roll angle is calculated using the following equation:

$=\sin ^{-1}\left(-a_{\mathrm{y}} / g \cos \theta\right) \rightarrow(2)$

Where $a_{\mathrm{y}}$ is the antero-posterior direction acceleration and $g$ is the gravity.

The trunk motion was described by the vector magnitude of acceleration. The upper trunk movement increased 3D acceleration Vector Magnitude (VM) of the wearable sensor. The vector magnitude was computed using the Equation 3.

$V M=\sqrt{ } a_{\mathrm{x}}{ }^{2}+a_{\mathrm{y}}{ }^{2}+a_{\mathrm{z}}{ }^{2} \rightarrow(3)$

Where $a_{\mathrm{x}}, a_{\mathrm{y}}$ and $a_{\mathrm{z}}$ are the lateral-, vertical-, and anterior direction accelerations, respectively. The mean of VM (mVM) and the standard deviation of VM (sdVM) were computed to measure dynamic movements of the upper trunk.

\section{Classification model with imbalanced physical activity data}

The four upper trunk motion features (i.e., roll, pitch, mVM, and sdVM) were applied to develop the seafarers' physical activity classification models. To consider their unstable living condition, two models (i.e. with and without the STS detection) were developed. As shown in Figure 1, the 3D-acceleration showed the characteristics of each physical activity. Lying and walking activities showed apparently different patterns, whereas sitting and standing activities generated similar patterns each other. The misclassification of sitting and standing activities has been typical issues in sedentary behavior assessment using the wearable sensors (i.e., actigraph) [10-12].

The first simple classification model without the STS detection was trained using only four main physical activities (i.e. lying, sitting, standing, and walking). The decision tree classification was applied to the first classification model. Equally balanced training data was utilized for the training session. In the decision tree training, a decision tree is used as a predictive model. Branches in the decision tree model represented the 
combinations of upper trunk motion features that lead to those leaves, and the leaves represent physical activity classes [15].

In the second model, the STS event detection was chosen as an indicator of the transition of the sedentary behaviors regardless of the directions of the STS. The second classification model classified four main physical activities (i.e. lying, sitting, standing, and walking) and the STS events (i.e., STS and nonSTS) in parallel. The STS event detection was used to improve the classification accuracy of the sedentary activities. As shown in the Table 2, the total STS event duration was less than 10 of 900 s (i.e. roughly $1 \%$ of total duration) on average. The imbalanced training data of the STS event was a key while training STS events. The Euclidean distances-based outlier clustering algorithm [16,17] was applied for the classification of the STS events from the dominant non-STS events. For the supervised anomaly event detection, non-STS activities were trained first and applied to classify STS events [18]. The five activities (i.e. including lying, sitting, standing, STS, and walking) were re-labeled either STS or non-STS so that STS could be considered as the anomaly events compared to all other non-STS labels. The outcomes of this process were used to modify uncertain sedentary activities. The strategy of the sedentary activity modification algorithm is summarized below:

Step 1) Training criteria of four physical activities by the decision tree model, and then go to Step 2

Step 2) Training only STS event criteria by labeling activity either STS or non-STS, then go to Step 3

Step 3) Classifying activity using trained criteria

Step 4) If lying or walking, determine an activity as it is, then go to Step 3 Else if sitting or standing activity, then go to Step 5

Step 5) Keeping a dominant activity and change a label as an unknown until the next STS, then Step 6

Step 6) Updating all unknown activity with the dominant static activity so far, then go to Step 3

Steps 5) and 6) improved classification accuracy by correcting any misidentified static activity using STS events.

\section{Results}

Among three static activities (i.e. lying, sitting, and standing), lying activity apparently differed from other two static activities. Walking also generated higher acceleration variations during the experiment. The four STS events (i.e., fast sit-to-stand, fast stand-to-sit, slow sit-to-stand, and slow stand-to-sit) showed the distinctive acceleration patterns from three static activities, while walking and the STS events showed the similar acceleration deviation.

\section{STS event detection results}

The STS events were trained after applying binary activity labels, such as STS and non-STS. Two fast STS events including fast sit-to-stand and fast stand-to-sit transitions were all correctly detected, whereas 2 of 38 slow STS events (i.e., slow sit-to-stand and slow stand-to-sit) were misclassified as walking. The misidentified slow STS events occurred when the subject was bent down to move forward at the beginning of the slow STS events. The upper body angle of the slow STS events generated similar upper body angles to sitting. Table 3 summarizes the STS event detection accuracy by proposed approach.

The efficacy of using the STS detection was evaluated by comparing the two models. Both models accurately classified lying and walking activities. The Non-STS model significantly misclassified the standing activity. $35.8 \%$ of standing activity was misclassified as sitting since the acceleration patterns of both sitting and standing were not clearly distinguished. The STS-based model substantially improved the classification accuracy of two static activities by taking the majority prediction vote in the case of unclear static activity labels. The prediction accuracy of sitting and standing of the STS-based model were $97.3 \%$ and $94.7 \%$, respectively (Table 4 ).

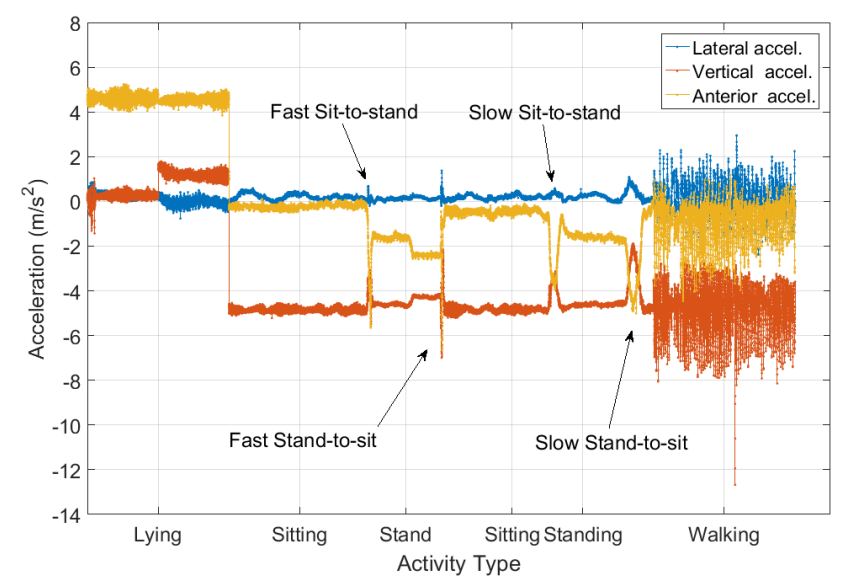

Figure 1. Acceleration patterns of five physical activities including lying, sitting, standing, STS, and walking.

Table 3. STS event detection accuracy for two tempos and directions using anomaly detection algorithm.

\begin{tabular}{lll}
\hline Direction & Tempo & Accuracy (\%) \\
\hline Sit-to-stand & Fast & 100 \\
\cline { 2 - 3 } & Slow & 94.7 \\
\hline Stand-to-sit & Fast & 100 \\
\cline { 2 - 3 } & Slow & 94.7 \\
\hline
\end{tabular}

Table 4. Seafarer physical activity classification results comparison between Non-STS model and STS-based model.

\begin{tabular}{llllll}
\hline \multirow{2}{*}{ Model } & True activity & \multicolumn{4}{c}{ Correct activity } \\
\cline { 3 - 6 } & & Lying & Sitting & Standing & Walking \\
\hline \multirow{2}{*}{ Non-STS model } & Lying & 100 & 0 & 0 & 0 \\
\cline { 2 - 6 } & Sitting & 0 & 88.6 & 9.4 & 2 \\
\hline
\end{tabular}




\begin{tabular}{llllll}
\cline { 2 - 6 } & Standing & 0 & 35.8 & 59.2 & 4 \\
\cline { 2 - 6 } Walking & 0 & 2.2 & 3.2 & 94.6 \\
\hline STS-based model & Lying & 100 & 0 & 0 & 0 \\
\cline { 2 - 6 } & Sitting & 0 & 97.3 & 2.2 & 0.5 \\
\cline { 2 - 6 } Standing & 0 & 3.9 & 95.7 & 0.4 \\
\cline { 2 - 5 } Walking & 0 & 2.2 & 3.2 & 94.6 \\
\hline
\end{tabular}

\section{Discussions and Conclusion}

We developed two classification models to compare the efficacy of the STS detection in the improvement of seafarer physical activity classification in the unstable living environment. The non-STS model is likely to misclassify between the two sedentary activities; sitting and standing. Grouping the number of sedentary activities by using the STS event greatly improved the classification accuracy on sitting and standing activities. The anomaly event detection approach to training the imbalanced data accurately recognized the different tempos (i.e. fast STS and slow STS) and the directions (i.e. sitting-to-stand or stand-to-sit) of the STS events. The results of the experimental study clearly supported that it was feasible to accurately monitor the mariner's prevalent physical inactivity during a sea voyage.

Although the outcomes of the proposed model were promising, the results were limited to a given scenario. The STS-based unknown data enhancing process is desired to consider as representative physical activity pattern recognition models in a real-world setting. Also, the STS direction recognition such as sitting-to-stand and stand-to-sit needs to be investigated further to improve the proposed models.

\section{References}

1. Scovill SM, Roberts TK, McCarty DJ. Health characteristics of inland waterway merchant marine captains and pilots. Occupational Medicine 2012; 62: 638-641.

2. Hjarnoe L, Leppin A. Health promotion in the Danish maritime setting: challenges and possibilities for changing lifestyle behavior and health among seafarers. BMC Public Health 2013; 13: 1165.

3. Xia SY, Zhang K, Shang W, Xing M, Chen L. Psychological and physiological effects of a long voyage on female seamen in China. Int J Clin Exp Med 2016; 9: 7314-7321.

4. Bevil CA, OConnor PC, Mattoon PM. Leisure activity, life satisfaction, and perceived health status in older adults. Gerontol Geriatr Edu 1994; 14: 3-19.

5. Heus R, Wertheim AH, Havenith G. Human energy expenditure when walking on a moving platform. Eur J Appl Physiol Occup Physiol 1998; 77: 388-394.
6. Oldenburg M, Harth V, Jensen HJ. Overview and prospect: food and nutrition of seafarers on merchant ships. Int Marit Health 2013; 64: 191-194.

7. Lefkowitz RY, Slade MD, Redlich CA. Injury, illness, and work restriction in merchant seafarers. Am J Industr Med 2015; 58: 688-696.

8. Hjarnoe L, Leppin A. Health promotion in the Danish maritime setting: challenges and possibilities for changing lifestyle behavior and health among seafarers. BMC Public Health 2013; 13: 1165.

9. Lawther A, Griffin MJ. The motion of a ship at sea and the consequent motion sickness amongst passengers. Ergonomics 1986; 29: 535-552.

10. Banerjee T, Skubic M, Keller JM, Abbott C. Sit-to-stand measurement for in-home monitoring using voxel analysis. IEEE J Biomed Health Inform 2014; 18: 1502-1509.

11. Santos LA, Santin-Medeiros F, Cardon G, Torres-Luque G, Bailon R, Bergmeir C, Garatachea N. Actigraph GT3X: validation and determination of physical activity intensity cut points. Int J Sports Med 2013; 34: 975-982.

12. Vanderloo LM, Di Cristofaro NA, Proudfoot NA, Tucker P, Timmons BW. Comparing the actical and actigraph approach to measuring young children's physical activity levels and sedentary time. Pediatr Exerc Sci 2016; 28: 133-142.

13. Shimmer3 sensor specification. Retrieved February 16, 2017, from http://www.shimmersensing.com/

14. Eric TH, Xu L, Hrovat D. Estimation of land vehicle roll and pitch angles. Vehicle System Dynamics 2007; 45: 433-443.

15. Aguiar B, Silva J, Rocha T, Carneiro S, Sousa I. Monitoring physical activity and energy expenditure with smartphones. Proc Biomed Health Inform 2014; 664-667.

16. Jiang MF, Tseng SS, Su CM. Two-phase clustering process for outliers detection. Patt Recogn Lett 2001; 22: 691-700.

17. Akoglu L, Tong H, Koutra D. Graph-based anomaly detection and description: a survey. Data Min Knowl Discov 2015; 29: 626-688.

18. Ramaswamy S, Rastogi R, Shim K. Efficient algorithms for mining outliers from large data sets. Proc ACM Sigmod $2000 ; 427-438$.

\section{*Correspondence to}

Chol-Seong Kim

Division of International Maritime Transportation Science

Collage of Maritime Affair

Jeollanam-do

Republic of Korea 